Gynäk. Rdsch. 1980;20(Suppl. 2):I-VI

\title{
Contents, Vol. 20, Supplement 2, 1980
}

\section{Editorial} 1

Gitsch, E.: Begrüssung und Eröffnung der Tagung 3

I. Hauptthema: Benigne Erkrankungen der weiblichen Brust Referat

Kucera, H.: KJinik der gutartigen Brustdrüsenerkrankungen

Koreferate

Holzner, J.H.: Die gutartigen Tumoren und tumorartigen Erkrankungen der weibli chen Brustdrüse

Kratochwil, A.; Dadak, C. und Bernaschek, G.: Ultraschalluntersuchung der weibli chen Brust 18

Wolf, G.: Mammadiagnostik der gutartigen Mammatumoren mittels Mammogramm 26

Kubista, E.: Plattenthermographie bei den Erkrankungen der weiblichen Brust ... 33 Schmid, R.: Organisation und Ergebnisse der Brustambulanz an der I. UniversitätsFrauenklinik Wien 39

Freilinger, G.: Chirurgische Aspekte benigner Brustdrüsenerkrankungen 46 Freie Vorträge

Euller, A.; Kemeter, P.; Feichtinger, W. und Nasr, F.: Möglichkeit der hormonellen Therapie gutartiger Brustdrüsenerkrankungen 58

Gerstner, G. und Wagner, G.: Bromocriptin - eine Alternative in der Behandlung der Mastitis puerperalis 64

Jerabek, R.; Dapunt, O.; Scharf, O. und Ortner, A.: Beitrag zur Mastitis nonpuerperalis 67

Terhpfer, H. und Euller, A.: Die Mastodynie aus psychosomatischer Sicht 72 Inhalt

II. Hauptthema: Chemotherapie und TherapiekontroIle beim Ovarialkarzinom Referat

Pickel, H. und Lahousen, M.: Chemotherapie des Ovarialkarzinoms 77

Koreferate

Lepuschütz, H.; Sager, W.D. und Pickel, H.: Computertomographische Therapiekontrolle beim Ovarialkarzinom 84

Urdl, W.; Pickel, H.; Pürstner, P. und Schrödl, P.: CEA - Ein Verlaufsparameter bei der Chemotherapie des Ovarialkarzinoms $\quad 90$

Janisch, H. und Szalay, S.: Indikation zur «second-look»-Operation beim Ovarialkar zinom 98

Burghardt, E.: Second-Look-Operation nach zytostatischer Therapie des Ovarialkrebses 103

Vorträge

Breitenecker, G.: Mortalität und Morbidität des Ovarialkarzinoms in Österreich . 109 
Holzer, E. und Lahousen, M.: Behandlung des Ovarialkarzinoms im Stadium la . . 113 Kupka, S. und Gitsch, E.: Ergebnisse der operativen Behandlung des Ovarialkarzi noms 118

Stummvoll, W.; Nagl, F. und Prestel, A.: Das fortgeschrittene Ovarialkarzinom: Diagnostik, Therapie und Ergebnisse am Bezirkskrankenhaus Ried im Innkreis 123

Leodolter, S.; Philipp, K. und Salzer, H.: Einsatz der Knochenszintigraphie beim Ovarialkarzinom $\quad 127$

Grünberger, V.; Dobrovits, H. und Hoffelner, L.: Palliativ- und Second-Look-Operationen beim Ovarialkarzinom 131

Szepesi, T.; Seitz, W.; Kogelnik, H.D. und Reinartz, G.: Simultane Radio-Chemo-

Therapie beim fortgeschrittenen epithelialen Ovarialkarzinom. Ergebnisse 1977-1980 135

Tatra, G. und Ludwig, H.: Fetale und plazentare Proteine beim Ovarialkarzinom . 138 Szalay, S.; Tatra, G. und Janisch, H.: Erste Erfahrungen mit dem «Cancer-SerumIndex» bei gynäkologischen Malignomen 142

Salzer, H.; Szalay, S.; Steiner, I. und Washüttl, J.: Einfluss von Adriamycin und Zyklophosphamid auf den Zellstoffwechsel beim Ovarialkarzinom in der Organkultur 144

Szalay, S.; Frimmel, H.; Bartl, W.; Breitenecker, G.; Denk, H. und Janisch, H.: Karzinoembryonales Antigen (CEA) und Blutgruppenantigene A, B in Paraffinschnitten epithelialer Ovarialtumoren $\quad 148$

Inhalt

HI. Hauptthema: Fortschritte in der gynäkologischen Urologie

Referat

Manzl, J. und Hetzel, H.: Die Bedeutung der Urodynamik in der Gynäkologie ... 151

Koreferat

Madersbacher, H.: Die Erfassung funktionell-neurogener Blasenentleerungsstörungen aus der Sicht des Urologen 161

Vorträge

Hetzel, H.; Manzl, J. und Dapunt, O.: Röntgenologische und urodynamische Untersuchungsergebnisse vor und nach Schlingenoperation 173

Mayer, H.G.K. und Klug, P.W.: Schlingenplastik mit Streifen aus der Fascia lata . 177

Nürnberger, N.: Endourethrale submuköse Tefloninjektion als Therapie bei Stressinkontinenz 181

Lahodny, J.: Anatomische und funktionelle Ursachen der weiblichen Belastungsinkontinenz 186

Pflüger, H.; Nürnberger, N.; Kupka, S.; Leodolter, S. und Wagner, G.: Diagnostik und Therapie postoperativer Blasenentleerungsstörungen nach gynäkologischer Ra-

dikaloperation 191

Salzer, H. und Wagner, G.: Die chronische Paraurethritis - ein seltenes Krankheitsbild? 195

Wagner, G. und Salzer, H.: Urethroskopie in der urodynamischen Diagnostik ... 199 Grünberger, W. und Tulzer, H.: Zur Therapie der postoperativen Harnverhaltung . 203 Bernaschek, G. und Kratochwil, A.: Sonographische Methode zur Messung des hinteren Urethrovesikalwinkels 208 
Riss, P.; Spernol, R. und Endler, M,: Erste Erfahrungen mit einem neuen Fragebogen im Rahmen der präoperativen Inkontinenzdiagnostik

212

Freie Vorträge über Geburtshilfe

Friedrich, F.; Kemeter, P.; Breitenecker, G. und Salzer, H.: Serumprogesteronwerte bei der normalen und bei der komplizierten Frühschwangerschaft 217

Mayer, H.O. und Stünzner, D.: Bedeutung von Infektionskrankheiten während der Schwangerschaft mit besonderer Berücksichtigung gestörter Schwangerschaften 220

Pavelka, R.; Riss, P. und Reinold, E.: Vaginalflora in einem Frühgeburtenrisikokollektiv 223

Ortner, A. und Manzl, J.: Management fraglicher Rötelninfektion in der Schwanger schaft und Rötelnprophylaxe durch den Gynäkologen

Brabec, W. und Dapunt, O.: Vergleich der tokolytischen Wirksamkeit von Hexoprenalin und Ritodrine bei prostaglandininduzierten Wehen 230

Elsner-Mackay. P. und Neumann, H.: Umgestaltung einer geburtshilflichen Abteilung im psychosomatischen Sinn 235

Bichler, A.: Gedanken über die sanfte Geburt 239

Inhalt

Weiss, P.A.M. und Winter, R.: Der Insulinbedarf der Diabetikerin im Verlauf der

Schwangerschaft 242

Rosanelli, K. und Rosegger, H.: Die Bedeutung des missfárbigen Fruchtwassers fur die Prognose des Neugeborenen 246

Winter, R.: Präpartale Diagnose von Missbildungen 250

Lichtenegger, W.; Sager, W.D. und Becker, H.: Fetale Missbildungsdiagnostik mit Ultraschall und Computertomographie 254

Hofmann, H.; Winter, R. und Pürstner, P.: Geburtsleitung bei Fusslage

Haller, H. und Golob, E.: Infektionsprophylaxe nach Kaiserschnitt

Schurz, A.R. und Ludescher, E.: Das Stillproblem in Vorarlberg 264

Schurz, A.R.: Infektmorbidität bei Wöchnerinnen und Neugeborenen bei Roomingin 266

Freie Vorträge über Gynäkologie

Lechner, W.; Battista, H.J. und Dienstl, F.: Untersuchungen zum Bleigehalt in der

Muttermilch in verkehrsreichen und verkehrsarmen Gegenden Tirols 268

Lang, E.: Klinisch-therapeutische Aspekte der anaeroben Infektionen in der Gynäko logie und Geburtshilfe271

Gerstner, G.; Huber, J.; Kofler, E. und Rotter, M.: Anaerobierinfektionen in der

Geburtshilfe und Gynäkologie 274

Kofler, E.; Gerstner, G. und Janisch, C.P.: Metronidazolprophylaxe bei gynäkologischen Operationen 277

Schurz, A.R.; Breitfellner, G. und Kiesler, J.: Der Candidanachweis. Wertigkeit verschiedener Methoden unter besonderer Berücksichtigung rehydrierter Teststreifen279

Philipp, K. und Brehm, R.: Ergebnisse der selektiven Thromboseprophylaxe mit Heparin bei gynäkologischen Operationen 281

Ruttensteiner, J. und Golob, E.: Laparoskopische Abklärung entzündlicher Adnexerkrankungen 285 
Huber, A.: Standard- und Mini-Gravigard bei Nulliparen. Vergleich der Ergebnisse zweier multizentrischer Studien $\quad 288$ 